\title{
The Future of the Chemical Sciences
}

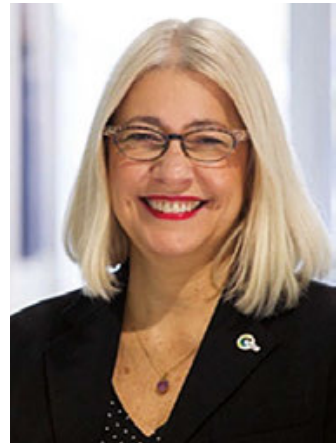

Preparing for an Uncertain Future

\section{by Alejandra Palermo}

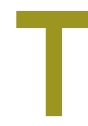

oday's world is characterised by turbulence with an increasing uncertainty in the global economy and in social and political relations. Scientific, social and technological trends are rapidly changing the way we live and work. These not only affect the nature and practice of chemistry, but also the roles that chemists play. With this in mind, the Royal Society of Chemistry launched the Future of the Chemical Sciences initiative to assess how the chemical sciences may evolve over the next ten to twenty years and the possible consequences for the community and society at large.

Science and technology almost always evolve in unpredictable ways, so extrapolating from the present what could happen in the future is likely to produce incorrect results. Thus, we used the scenario planning approach which considers multiple different "futures" to explore how emerging trends may evolve. By considering several plausible futures affecting our discipline, we can be equipped to adapt to and, where possible, influence them. In a nutshell "it is better to prepare for the future rather than to predict it."

Over the course of a year, we put together a multistage programme and worked with leaders from around the world and across different sectors to explore ways in which the chemical sciences might evolve. Our approach was an iterative method of consultation, analysis, synthesis, and validation, starting with a consultation process with key opinion leaders about the future. During the initial stages, we identified seven themes that might influence the future of chemistry.

\section{Role of the chemical sciences-essential and connected}

Increasing interdisciplinarity between chemistry and other sciences, most notably biology, is essential to tackling major challenges and drives new approaches to research. The challenges posed by interdisciplinarity must be addressed with increased urgency if chemistry is to retain its prominence as an influential subject. Chemistry might become a "follower" whilst other sciences lead on discoveries.

\section{Future demand- \\ chemistry for impact \\ The world faces major challenges demanding work} in partnership between countries, disciplines, and the public and private sectors. Health, climate change and energy, water and food production, and infrastructure are key sectors where chemistry could make a critical difference. However, the role of chemistry is not well understood by policymakers, funders, and even the chemistry community itself.

\section{Globalisation-collaboration and competition}

The chemical sciences are a global endeavour and the UK is recognised as a leading scientific country. Capitalising on this remains an issue. International collaboration is essential to tackling global challenges-UK needs to secure its role in future global partnerships.

\section{Technology-efficiency and innovation}

Technology will drive radical improvements. This will further impel interdisciplinary working, changing the nature of research, the character of academic and professional careers, and industrial structures.

\section{Open-disruptive, inevitable and uncertain}

The general trend towards "open" will be disruptive. Open data, access, and content will lead to structural changes though actual outcomes are unclear.

\section{Funding structures, institutions and education-the need for change}

There is an imperative need for funding mechanisms and institutions to adapt to this new world. Improvements in technology and automation would significantly impact research.

\section{Social trends-changing workforce and public} attitudes

An aging population will impact the workforce. Changing demographics and an increasingly mobile workforce could trigger a war for talent. The chemistry sector must strive to attract people from diverse backgrounds and achieve gender balance. 
Building on these themes, we identified a range of 'weak signals'-drivers that could become important catalysers of change, developing case studies for each. These were used during the scenario planning workshops leading to the development of 4 plausible scenarios set in the next 10-20 years.

\section{Scenario 1: Chemistry saves the world}

This is a world that has been shocked by the challenges facing society and has reacted accordingly. Multiple global challenges have focused research efforts and resources on solving these by bringing together different sectors in massive initiatives. Chemists are seen as leaders and can showcase areas where chemistry has successfully addressed global problems.

Chemistry now exists in the cultural space and is connected to the public in popular ways. Companies have a greater interest in chemistry. Non-traditional chemistry employers take chemists into their workforce. Funding for basic research becomes rarer-charities and philanthropists become more important. Discovery is more incremental.
Academics have multiple roles and multidisciplinarity is essential. A new qualification recognising impact and interdisciplinary emerges.

A flourishing ecosystem of chemistry start-ups exists, innovation in industry is linked to these. New forms and models of regulation emerge.

\section{Scenario 2: Push button chemistry}

This is a world shaped by the extraordinary developments in science and technology-where DIY culture has challenged current infrastructure. Laboratory equipment is easier to use and can be accessed remotely. Most lab time is rented and the majority of work is automated. Access to low-cost, on-demand experimentation and modelling has created a more entrepreneurial culture in chemistry. There is a large online community of people synthesising and sharing their own products.

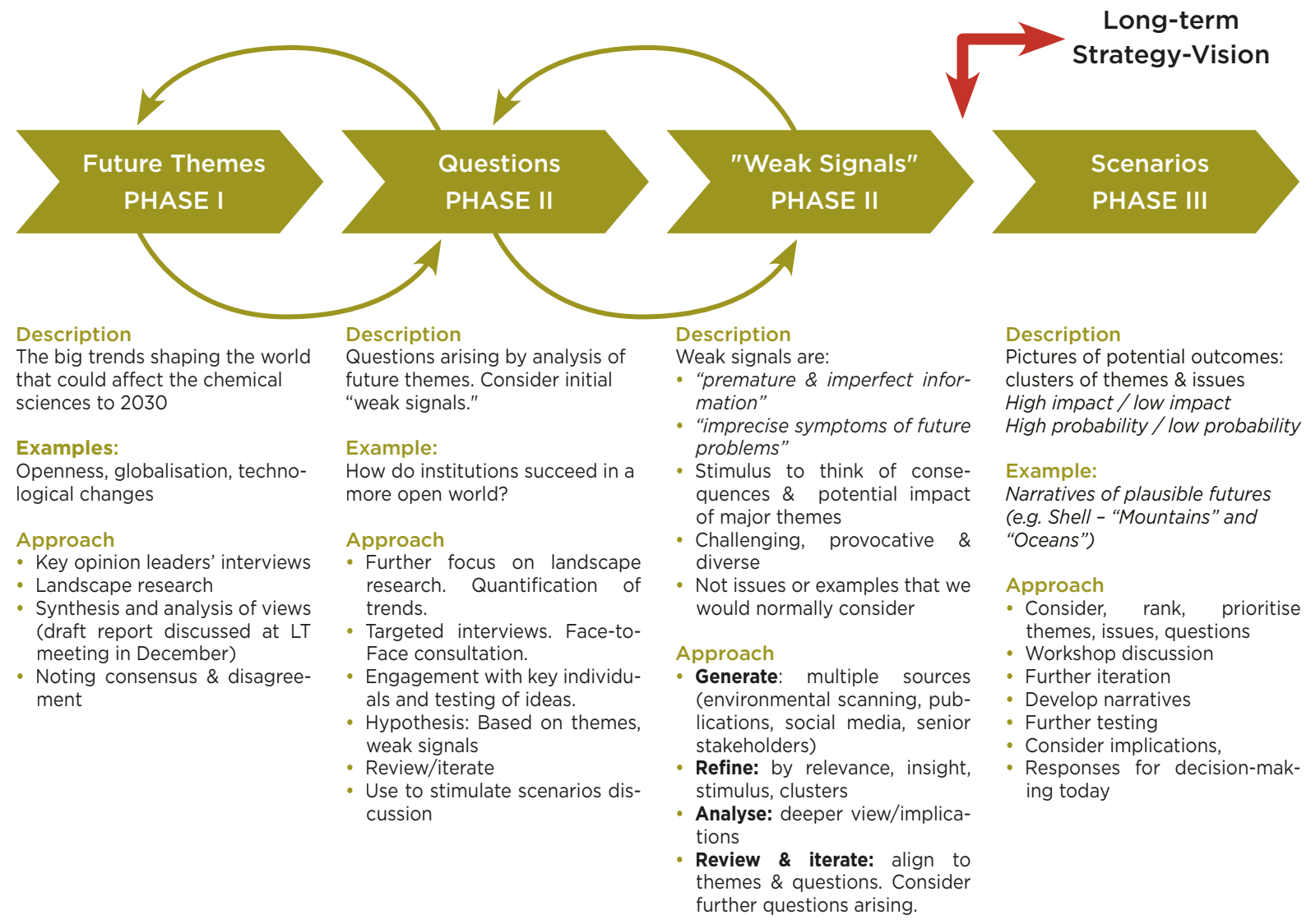


New crowdfunding mechanisms have emerged to exploit discoveries, challenging traditional industry.

Chemistry goes underground. The low cost and accessibility of experimentation has allowed a greater degree of personalisation in lifestyle and personal care chemical products. A world of push-button chemistry is increasingly difficult to regulate. There is an unregulated black market for advice on how to synthesise your own products. New models for quality assurance emerge, brands become more important.

Chemistry education is more entrepreneurial. A few global teaching institutions remain, offering virtual education. A chemist is likely to be self-employed.

\section{Scenario 3: A world without chemists}

This is a world where chemists have become increasingly less influential and productive, and other disciplines have taken the lead on discovery. The pace of this change was so fast that chemists could not reinvent themselves. Automation and advances in Al have replaced many traditional jobs, and creativity has not been embedded in the education of the current generation of chemists.

The identity of chemistry as a specific discipline has disappeared and has re-emerged as part of other subjects. The critical mass of chemists is dispersed into sub-groups. Chemistry has a fragmented role in academia.

The general public does not understand what chemistry is or its impact. Young people do not see chemistry as a viable career option; they are attracted to biology and physics, which appear to be making more exciting discoveries. The chemical industries are perceived to be in decline. No one wants to be a chemist.

Universities no longer offer specific 'chemistry' courses. Within two generations the skills required to teach chemistry are lost. The connections between the different aspects of chemistry are lost. There are no chemists, but many people work on chemistry.

The world is effective at producing new chemistry research, for a while. Eventually research hits a wall and there is no new basic research nor specific research funding for 'chemistry.'

\section{Scenario 4: Free market chemistry}

This is a world which is poor, shaped by war, climate change and many "local" crises. In response to

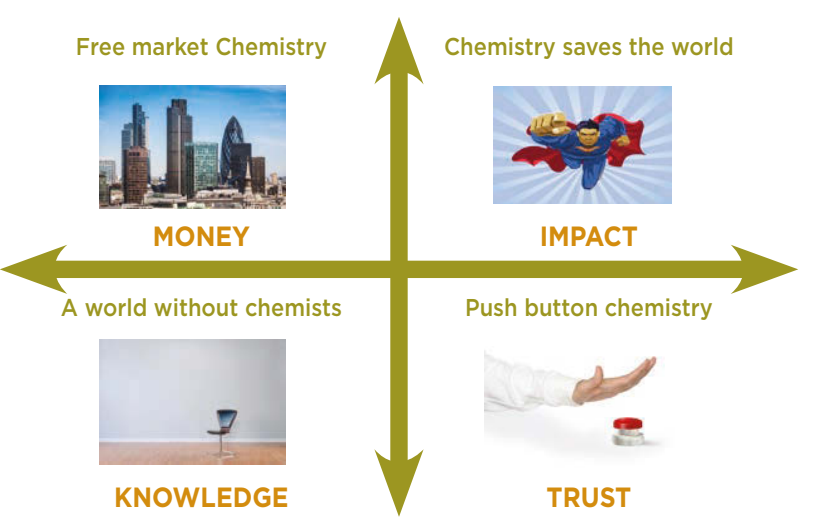

these, researchers frame what they study by problems.

There is now a market for private research. The consumers of science become the funders-a new philanthropy evolves. Chemistry departments have become private virtual institutes. There is less basic research in this world of 'problem solving.'

Education is disconnected from research. Many universities have closed, and the academic institutions that remain are focused on teaching. Teaching becomes more virtual and applied. The value of a PhD has decreased.

Chemists are no longer accountable to society. Chemistry is increasingly elitist and it is now important to know the right people to access funding.

\section{Next Steps}

We believe that the scenarios developed will challenge our community to move beyond conventional thinking and plan strategically for the future. We hope they provoke reflection on opportunities and challenges and stimulate discussion about how best to prepare for an uncertain future and the capabilities needed in the plausible scenarios. Embedding the scenarios should provide a rational way of thinking about the future and tackling difficult problems in which chemistry and chemists should expect to play a central role.

\section{See full report @ rsc.li/futurechem}

Check Alejandra Palermo's presentation at the WCLM2017 @ iupac.org/ project/2016-032-2-020 and see next feature article.

Alejandra Palermo <PalermoA@rsc.org> FRSC, Senior Manager, External Relations, Royal Society of Chemistry-is a chemical engineer with a PhD in Materials Science. Her independent academic career began as Assistant Professor in Chemical Engineering in Argentina, before joining Cambridge University under a Royal Society Visiting Fellowship. 